
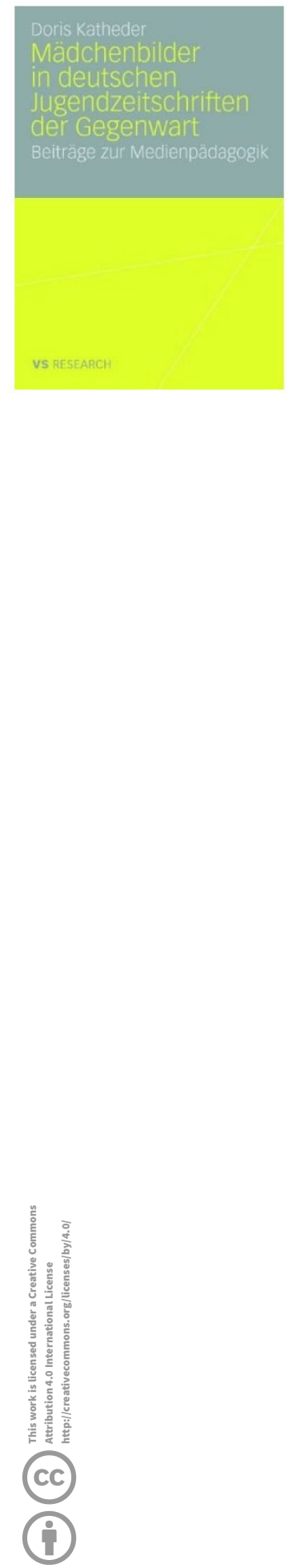

Doris Katheder

Mädchenbilder in deutschen Jugendzeitschriften der Gegenwart

Beiträge zur Medienpädagogik

Wiesbaden: VS, 2008. 347 Seiten

ISBN: 978-3-531-15940-9

$€$ 39.90; CHF 68.-

\section{Kleine Mädchenwelten statt große Weltmädchen}

Doris Katheder untersucht in ihrer Dissertationsschrift (PH Freiburg) die mediale Darstellung von jungen Frauen und die Inszenierung von Geschlechterverhältnissen in einem Medium, das bislang nie so recht akademische Aufmerksamkeit fand. Im Zentrum ihrer Ausführungen steht eine detaillierte Beschreibung der von ihr erarbeiteten qualitativen Empirie: In 60 Seiten „Einleitung“ führt die Autorin in die Fragestellung, methodische Grundlagen und den Forschungsstand ein; auf etwa 220 Seiten entfaltet sie sodann die (theoretisch flankierten) empirischen Ergebnisse, die in einen ca. 30seitigen „Schlussteil“ münden, der die wichtigsten Befunde zusammenfasst und verortet. Den Forschungsstand im engeren Sinne erarbeitet sie anhand der wenigen Untersuchungen, die explizit zu Mädchenbildern in Jugendzeitschriften vorliegen. Im Wesentlichen bestätigen diese, was Gender \& Media Studies in ähnlicher Weise für Werbung, Film, Serie und Musikvideoclip konstatieren: die Reduktion weiblicher Lebenswelten und Interessen auf den privaten Nahbereich, die romantische Liebe als Lebensziel und Ideologie, die Erotisierung des weiblichen Körpers, stereotype und konservative Körperbilder und Rollenverteilungen sowie ausgeprägte Hierarchien im Verhältnis von Weiblich- und Männlichkeit (vgl. insbes. 46ff.). In ihrer eigenen Untersuchung greift Doris Katheder auf die qualitative Inhaltsanalyse zurück. Aufgrund der in den Zeitschriften charakteristischen Text-Bild-Relationen ergänzt sie das Vorgehen um Hartmut Stöckls „linguistisch-semiotische Bildtheorie als methodische Herangehensweise“ (27). Daher lässt sich die Arbeit nicht nur inhaltlich auf die von den Zeitschriften gezeichneten Mädchenbilder hin lesen, sondern auch methodisch als Anwendung einer medienlinguistisch fundierten Text-Bild-Analyse (anschaulich etwa die Werbeanalysen 201ff.). Untersucht werden sowohl kommerzielle (z.B. ,Bravo Girl!', ,Brigitte 
Young Miss') als auch nicht-kommerzielle Mädchen- und Jugendzeitschriften. Bei den kommerziellen Angeboten konzentriert sich die Autorin auf die Foto(love)storys, die Beratungsrubriken und die Werbung als zentrale Verhandlungsorte der Hauptkategorie „Mädchen“.

Grundsätzlich bestätigt die kritisch-geschlechtersensible Forschungsperspektive die zuvor theoretisch konstatierten engen Grenzen der Rollenzuschreibungen, Schönheitsideale und Körperinszenierungen in den kommerziellen Blättern. In den teils gegenläufigen Orientierungen der Genres Fotostory, Werbung und Beratung changieren die Zeitschriften zwischen Rationalisierung, (Re-)Romantisierung und Realitätstranszendierung. Tenor der Arbeit ist es, die Reduktion der Lebenswelten, Interessen und Handlungsoptionen junger Frauen aufzuzeigen. Emanzipation findet allenfalls im Verborgenen und Privaten statt und bedeutet selten mehr als eine ästhetische Individualisierung und Selbststilisierung. Als charakteristisch, auch in Abgrenzung zu anderen Medienangeboten, darf die „romantische Provinzialisierung" in Fotostorys gelten (101ff.). Mädchenwelten sind hier oft Jugendzimmerwelten, die als "Gehäuse" von der Außenwelt abschirmen und den (vermeintlich weiblichen) Fantasien, Schwärmereien und Gefühlswogen ein ebenso sicheres wie entpolitisiertes, handlungsarmes Zuhause geben. Die Beratungsangebote nehmen Unsicherheiten in Beziehung und Liebe, Sexualität und Körperbild zwar auf, konterkarieren sich indes selbst, wenn sie zugleich Rationalität und Realismus einfordern, das Ideal romantischer Paarbeziehung überhöhen und auf prototypische Jungen- und Mädchenbilder rekurrieren. Nicht nur in der Werbung beteiligen sich die Zeitschriften zudem an der Konstitution rigider Schönheits- und Handlungsnormen, die ihren Beitrag leisten, dass auch in Zukunft weiter Beratungsbedarf besteht. Nun ließe sich vermuten, dass die Autorin die nicht-kommerziellen, „alternativen" Mädchenzeitschriften als Gegenhorizonte eines authentischen und unverstellten Blicks auf Mädchenwelten aufspannt. Trotz aller Sympathie enthält sie sich einer solchen Binarität und macht deutlich, wie die Mädchen und jungen Frauen einerseits kritisch Bezug nehmen auf die (medien-) kulturellen Stereotype und Narrationen und mit Konventionen brechen, andererseits aber auch selbst unhinterfragten, etwa biologisierenden Denkmustern verhaften bleiben.

"Mädchenbilder in deutschen Jugendzeitschriften“ ist denjenigen zu empfehlen, die sich kritisch mit Medienkultur und medialen Darstellungen von Geschlecht auseinandersetzen. Doris Katheders Fragen stellen sich für alle, die reflektieren, welche medialen Bausätze geschlechtsidentitärer Orientierung 
Kindern und Jugendlichen vorgesetzt werden. Es sind dabei weniger die bekannten (gleichwohl berechtigten) Thesen der Asymmetrie und medialen Verkürzung, sondern der Facettenreichtum und die Detailliertheit der beschriebenen Phänomene, die den Reiz der Lektüre ausmachen. Der Fokus auf Jugendzeitschriften erscheint berechtigt in einer Medienlandschaft, in der Magazine wie ,Bravo', die vor nicht unendlicher Zeit selbst regelmäßig Ansto $B$ des Ärgers waren, angesichts vermeintlicher oder tatsächlicher Bedrohungen aus dem Internet und dem Computerspiel als nahezu unschuldig und mithin als Verbündete von Lehrer/innen und Eltern gelten. Was Jugendliche dann tatsächlich aus dem Material machen, wie sie sich dieses aneignen, bleibt allerdings einer Rezeptionsstudie vorbehalten, die mit Blick auf medienübergreifende Nutzungs- und Orientierungsmuster wohl kaum als reine Einzelmedienstudie zu realisieren wäre.

Doris Katheders Buch ist lesenswert und aufschlussreich, aber nicht immer in gleichem Maße zu lesen. Das Inhaltsverzeichnis wirkt, nicht zuletzt aufgrund mangelnder typografischer Absetzungen, überladen; vielerorts wären Paraphrasierungen und indirekte Belege anstelle der zahlreichen Wortgruppen- und Satzzitate dem Lesefluss dienlich. Diejenigen, die sich weniger akademisch-wissenschaftlich mit der Thematik beschäftigen möchten, seien darauf hingewiesen, dass die Qualifizierungsarbeit um wissenschaftliche Sprache bemüht ist. Das muss aber nicht entmutigen, bilden doch die detailreichen Fallanalysen und die durchweg farbigen Illustrierungen hierzu einen Gegenpol. Forschungsmethodisch regt die Arbeit zum weiteren Nachdenken über angemessene Formen sozialwissenschaftlicher Bildinterpretation an. Die bestehenden Ansätze unterscheiden sich insbesondere in der Frage, wie sie mit Kontextwissen umgehen, ob und an welcher Stelle des Analyseprozesses sie dieses einbinden. In Katheders Analysen ist die Kontextualisierung und Anreicherung mit Wissen aus der Fachliteratur allgegenwärtig. Die Grenze zwischen Medienproduktanalyse und Inferenz auf die Rezeption gerät - dem Rezensenten - dabei mitunter zu fluide, wenn beispielsweise farbpsychologische Wahrnehmungsthesen die Interpretation leiten (213f.). Zugespitzt darf gefragt werden, ob sich Essentialisierungen und Rationalisierungen, die in Bezug auf den Forschungsgegenstand (Mädchenbilder / Geschlecht) dekonstruiert werden sollen, auf diese Weise nicht unhinterfragt in die Methode der Bildinterpretation einschreiben. 